\title{
Macroporous hydrogel scaffolds with tunable physicochemical properties for tissue engineering constructed using renewable polysaccharides
}

\begin{abstract}
Xiaoliang $\mathrm{Qi}^{1}$, Ting $\mathrm{Su}^{2}$, Mengying Zhang ${ }^{2}$, Xianqin Tong, Wenhao $\mathrm{Pan}^{3}$, Qiankun Zeng ${ }^{2}$, Zaigang Zhou ${ }^{1}$, Liangliang Shen ${ }^{2}$, Xiaojun He ${ }^{1}$, Jianliang Shen ${ }^{1,2 *}$

${ }^{1}$ State Key Laboratory of Ophthalmology, Optometry and Vision Science, School of Ophthalmology and Optometry, School of Biomedical Engineering, Wenzhou Medical University, Xueyuan West Road, Lucheng District, Wenzhou 325027, China ${ }^{2}$ Wenzhou Institute, University of Chinese Academy of Sciences, Xinsan Road, Lonwan District, Wenzhou 325001, China

${ }^{3}$ Department of Orthodontics, School \& Hospital of Stomatology, Wenzhou Medical University, Xueyuan West Road, Lucheng District, Wenzhou 325027, China

*Address correspondence to shenj1@wibe.ac.cn (J. Shen).
\end{abstract}

Table S1. Compositional ratios utilized for the construction of salecan/א-carrageenan gels

\begin{tabular}{lllll}
\hline Ingredient & \multicolumn{2}{l}{ Designation } & & \\
\cline { 2 - 5 } & $\mathrm{G} 1$ & $\mathrm{G} 2$ & $\mathrm{G} 3$ & $\mathrm{G} 4$ \\
\hline Salecan $(2 \%, \mathrm{w} / \mathrm{v})(\mathrm{mL})$ & 0 & 1 & 3 & 5 \\
$\kappa$-carrageenan $(2 \%, \mathrm{w} / \mathrm{v})(\mathrm{mL})$ & 10 & 9 & 7 & 5 \\
\hline
\end{tabular}




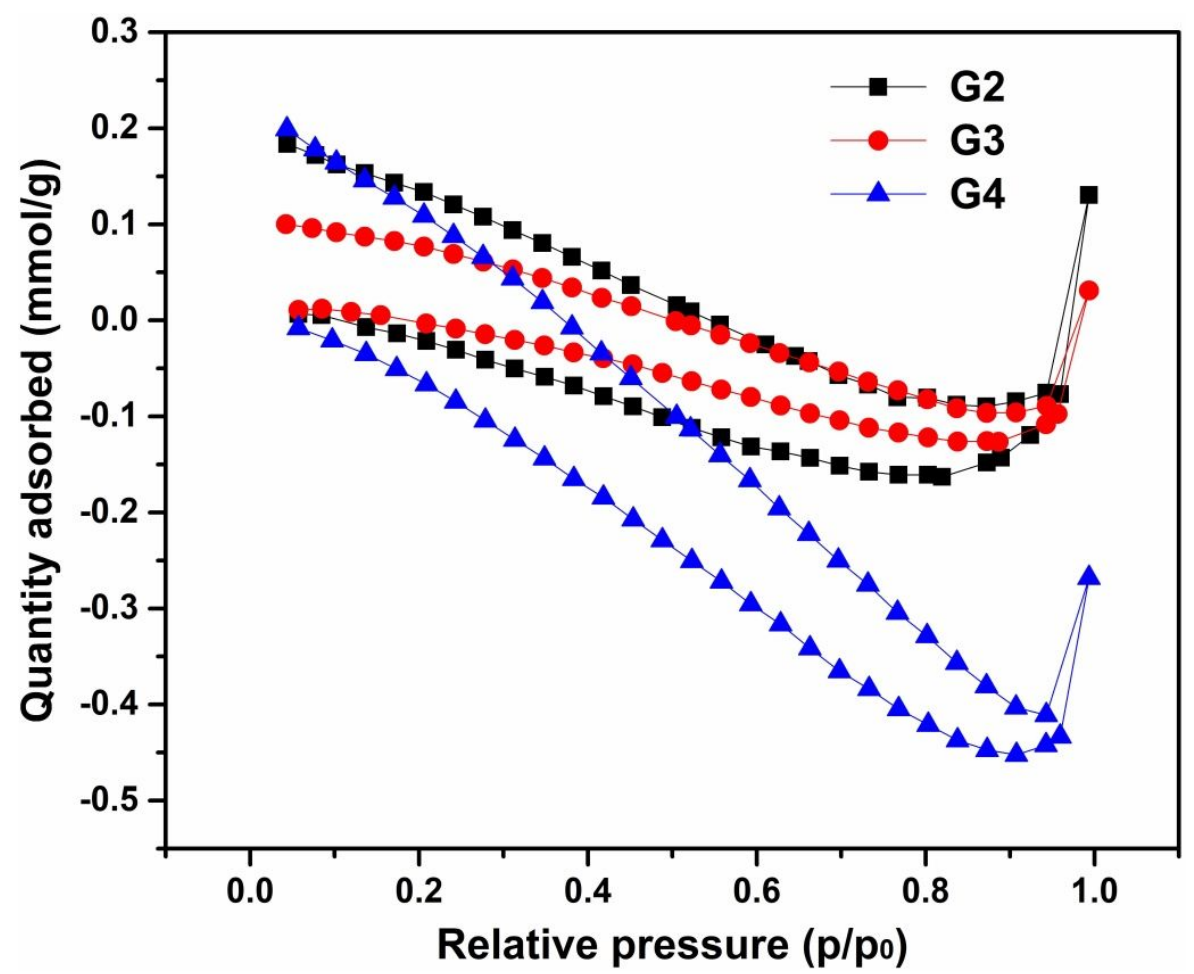

Figure S1. $\mathrm{N}_{2}$ adsorption-desorption isotherms of salecan/א-carrageenan hydrogels (G2, G3 and G4). 